\title{
Application of drought and salt stress can improve tomato fruit quality without jeopardising production
}

\author{
B.A.E. Van de Wal ${ }^{1, a}$, L. Van Meulebroek ${ }^{2}$ and K. Steppe ${ }^{1}$ \\ ${ }^{1}$ Laboratory of Plant Ecology, Faculty of Bioscience Engineering, Ghent University, Ghent, Belgium; ${ }^{2}$ Laboratory of \\ Chemical Analysis, Faculty of Veterinary Medicine, Ghent University, Ghent, Belgium
}

\begin{abstract}
High-tech tomato greenhouse systems, which are the standard in Northern Europe (especially in Belgium and the Netherlands), mainly aim for high yields, up to $60 \mathrm{~kg} \mathrm{~m}^{-2}$. However, quality should be considered equally important, as consumers are willing to pay higher prices per $\mathrm{kg}$ for a high quality product. Influencing the plant water status is acknowledged to strongly influence fruit quality, as water deficit or increased salinity may result in higher dry matter content, a main determinant of tomato quality. Unfortunately, this increase in quality is often associated with a decrease in fresh yield, making a thorough insight on the controlling factors of both aspects critical if one aspires to optimise the final product value. The objective of the current research was therefore to combine plant water status monitoring with the assessment of an array of fruit quality parameters and yield, for both drought and salinity treatments in order to further clarify this interrelationship. To this end, we set up an experiment in a controlled greenhouse environment, where tomato plants (Solanum lycopersicum 'Dirk') were exposed to four different treatments: control, drought stress, and two levels of salt stress (EC levels of 4 and $6 \mathrm{dS} \mathrm{cm}^{-1}$ ). Plant water status was monitored by measuring stem water potential. Furthermore, fruit yield, as well as a set of fruit quality parameters (hexose sugars content, organic acids content, and firmness) was evaluated. Results showed that fruit quality does benefit from both drought and increased salinity, and that the highest salinity level scored the best on all measured quality aspects. Moreover, we observed that even small water deficits, induced either by mild drought or salt stress, improved fruit quality, without jeopardising yield. The acquired insights, combined with mechanistic modelling, may ultimately lead to a more efficient greenhouse management with higher quality tomatoes.
\end{abstract}

Keywords: stem water potential, glucose, fructose, malic acid, citric acid, firmness, electrical conductivity

\section{INTRODUCTION}

High production is the main concern for most tomato growers in high-tech greenhouses, which are the standard in Northern Europe (especially Belgium and the Netherlands). However, since these tomatoes are produced mainly for the fresh market, fruit quality is equally important, as consumers are increasingly demanding. In this regard, quality is determined by a variety of factors, such as appearance (colour, shape, size), texture, firmness, aroma, and taste (Dorais et al., 2001). The latter is strongly related to the amount of reducing sugars and organic acids in the fruit, and especially to their interrelationship (Ke and Boersig, 1996; Auerswald et al., 1999; Dorais et al., 2001). This is rather evident since these two component classes represent approximately 50\% (Davies and Hobson, 1981) and 10-13\% (Ho and Hewitt, 1986) of the total dry matter, respectively.

\footnotetext{
a E-mail: bart.vandewal@UGent.be
} 
Both production (fresh weight) and quality (dry weight percentage) are influenced by an array of environmental factors. For example, high light intensities have been demonstrated to improve dry matter content through an increase in available photoassimilates (Bertin et al., 2000; Dorais et al., 2001; Anza et al., 2006, Hanssens et al., 2015). Vapour pressure deficit (VPD) is another factor known to affect fruit dry matter content, since high VPDs increase fruit transpiration and decrease xylem influx, while phloem influx is hardly influenced (Guichard et al., 2005). This leads to a relatively higher influx of assimilates in comparison to water and thus to a higher dry matter content. Finally, water availability is another crucial factor with a major influence on water and dry matter accumulation in the fruit. While water deficit (whether induced by drought or increased salinity) generally leads to higher dry matter contents, and sugar and acid concentrations (Mizrahi, 1982; Mitchell et al., 1991; Veit-Köhler et al., 1999; Plaut et al., 2004), this is mostly due to a decrease in water influx and thus fresh weight. This means a loss of revenue for the grower and is hence unwanted.

Water deficit due to drought or due to increased salinity (electric conductivity, EC) are known to impact the fruit water and carbon balance differently (Mitchell et al., 1991). However, these particular conditions have not often been studied simultaneously (Mitchell et al., 1991; Plaut et al., 2004). Therefore, in this study we aimed at clarifying these differences by comparing an array of fruit quality parameters as well as fruit production for a drought treatment and two increased salinity levels. As such, it was envisaged to deepen the knowledge on the trade-off between fruit production and fruit quality.

\section{MATERIALS AND METHODS}

Tomato plants (Solanum lycopersicum 'Dirk') were grown in a $60 \mathrm{~m}^{2}$ glasshouse compartment of the Institute for Agricultural and Fisheries Research (ILVO) in Melle, Belgium. Plants were sown in rockwool blocks (Grodan Delta, Grodan, Roermond, the Netherlands) on July 3, 2014. These blocks were transplanted onto rockwool slabs (Grodan Vital, Grodan, Roermond, the Netherlands) on September 2, 2014, which also represented the start date of the four different water treatments. The experiment was ended on December 16, 2014, at the moment that 7 trusses per plant had been harvested.

A trickle irrigation system provided the plants with nutrient solution every 90 minutes, or when the radiation sum exceeded $2 \mathrm{MJ} \mathrm{m}^{-2}$, which are commonly used values in commercial practice. Besides a control group (henceforth denoted as $\mathrm{C}$ ), which received ample irrigation (i.e. 30-50\% drain) at an EC level of $2.7 \mathrm{dS} \mathrm{m}^{-1}$, there was a drought group (D), receiving only $75 \%$ of the control irrigation amount, and two different salt stress groups (EC4 and EC6), receiving ample irrigation, but with EC levels of $4.0 \mathrm{dS} \mathrm{m}^{-1}$ and $6.0 \mathrm{dS} \mathrm{m}^{-1}$, respectively. Increased EC was achieved by adding a $2: 1 \mathrm{NaCl} / \mathrm{CaCl}_{2}$ solution to the nutrient solution. Each group comprised a gutter with nine plants, including two edge plants on which no measurements were done. At least one extra gutter at each side was filled with tomato plants to avoid any border effects.

\section{Manual plant and fruit measurements}

To assess the impact of the water treatments on the plants, stem xylem water potential $\left(\Psi_{\text {stem }}\right)$ was measured weekly on 3 plants per treatment, using a pressure chamber (Model 1000, PMS instruments, Albany, OR, USA). Given the destructive nature of these measurements, these plants were not used for fruit measurements, since leaf pruning might affect fruit quality and production. Fruit growth (diameter) was monitored using a calliper, and fruit fresh and dry weights were determined at harvest. Trusses were all pruned to five fruits per truss and harvested at the red-ripe stage. 


\section{Sugar and acid analyses}

From each truss, the third tomato was immediately frozen in liquid nitrogen at harvest, and stored at $-80^{\circ} \mathrm{C}$ for further analysis of sugars (fructose, glucose and sucrose) and acids (malic acid and citric acid).

Hexose-sugars were extracted from the ground fruit samples with $100 \%$ ethanol at $70^{\circ} \mathrm{C}$ for $10 \mathrm{~min}$, followed by $3 \mathrm{~h}$ at $45^{\circ} \mathrm{C}$ and centrifugation at $5000 \mathrm{~g}$ and $8^{\circ} \mathrm{C}$ for $10 \mathrm{~min}$. Chromatographic separation and detection was achieved using a Prevail Carbohydrate column $(250 \times 4.6 \mathrm{~mm}, 5 \mu \mathrm{m})$ (Grace Alltech, Deerfield Illinois, USA) and an Agilent $1100 \mathrm{High}$ Performance Liquid Chromatography (HPLC) system, coupled to an Alltech 3300 electrochemical light scattering detector (Grace Alltech, Deerfield, Illinois, USA).

Organic acids were extracted from the ground fruit samples with ultrapure water at $100^{\circ} \mathrm{C}$ for $15 \mathrm{~min}$, followed by centrifugation at $5000 \mathrm{~g}$ and $8^{\circ} \mathrm{C}$ for $10 \mathrm{~min}$. Next, samples were purified using $0.45 \mu \mathrm{m}$ filters (PP Syringe, Sigma Aldrich, Munich, Germany). Separation and detection was achieved using a GraceSmart Reverse Phase C18 column (150 × $2.1 \mathrm{~mm}, 5 \mu \mathrm{m})$ (Grace Davison Discovery Sciences, IL, USA) and an Accela 600 HPLC system coupled to an LTQ XL linear ion trap mass spectrometer (Thermo Fisher Scientific, San Jose, CA, USA).

\section{Fruit firmness analysis}

At harvest, the first and fourth fruit were taken from each truss and kept at a constant temperature of $20^{\circ} \mathrm{C}$ for $36 \mathrm{~h}$. Then, their firmness was measured using an A5942 Instron Texture Analyser (Instron, Norwood, MA, USA). Tomatoes were punctured to a depth of $10 \mathrm{~mm}$ at a speed of $1 \mathrm{~mm} \mathrm{~s}^{-1}$ with a cylindrical rod, having a diameter of $16.99 \mathrm{~mm}$.

\section{Statistical analyses}

One-way analysis of variance (ANOVA) was used to compare measured plant and fruit quality parameters between the four treatments. The Holm-Sidak multiple comparison method was then used as a post-hoc test to determine differences between individual groups. When normality criteria were not met (this was true for firmness and sucrose and malic acid concentrations), a Kruskal-Wallis ANOVA on ranks was used, followed by a Dunn's method posthoc test. All statistical analyses were performed in SigmaPlot 12.0 (Systat Software Inc., San Jose, CA, USA).

\section{RESULTS AND DISCUSSION}

\section{Plant water status}

Salt treatments had a significant effect on the plant water status, with $\Psi_{\text {stem }}$ of EC4 and EC6 differing $0.06 \mathrm{MPa}(28 \%)$ and $0.10 \mathrm{MPa}(47 \%)$ from the control treatment, respectively (Fig. 1). Given the fact that $\Psi_{\text {stem }}$ was not very negative overall due to the timing of the experiment (autumn), these differences clearly showed the effectiveness of the salinity treatments. On the other hand, the $\Psi_{\text {stem }}$ of the drought treatment did not differ significantly from the control. 


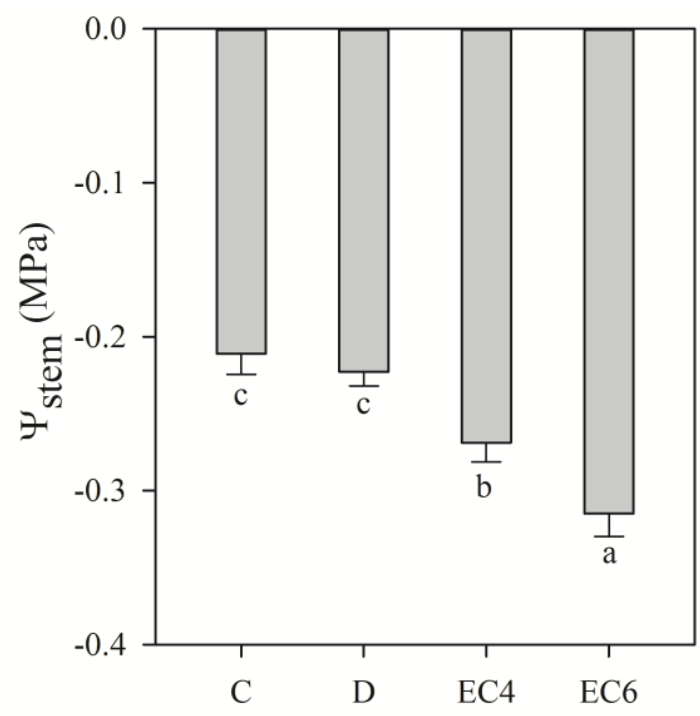

Figure 1. Mean stem water potential, measured between 12 and $1 P M( \pm S E)(n=24)$, for the different treatments (C - control, D - drought treatment, EC4 - salt treatment with an EC of $4 \mathrm{dS} \mathrm{m}^{-1}$, EC6 - salt treatment with an EC of $\left.6 \mathrm{dS} \mathrm{m}^{-1}\right)$. Significant differences $(P<$ $0.05)$ across bars are denoted by different letters.

\section{Fruit quality and production}

While plant water status was not noticeably affected by drought (Fig. 1), sugar concentration was (Fig. 2A). Both fructose and glucose levels were more than $50 \%$ higher than in $\mathrm{C}$ and comparable to $\mathrm{EC4}$, although the plant water status of the latter was more affected by its treatment (Fig. 1). As expected, EC6 showed even higher sugar concentrations, which were more than doubled compared to $\mathrm{C}$. While sucrose concentrations are typically only marginal at harvest (Ho and Hewitt, 1986), they were noticeably higher for EC6 (Fig. 2B). Since sugars comprise up to $50 \%$ of the total dry matter content (Davies and Hobson, 1981) and are crucial in consumers' taste appreciation, these higher concentrations strongly affect fruit quality. It should be noted, however, that the lower sugar concentration in C compared to the other treatments is mainly due to a dilution effect, as no significant differences were found between sugar amounts on a per fruit basis (data not shown). The fact that the total amount of sugars on a per fruit basis is neither higher nor lower along treatments also indicates that the different water regimes had no strong limiting effects on photosynthesis, or at least not to the extent that the amount of assimilates, transported to the fruits, was affected. This might have been compensated for by investing less assimilates into vegetative growth, since fruits are the strongest sinks during the generative phase (Ho and Hewitt, 1986).

A second major determinant of taste, and thus fruit quality, is the content of organic acids. Tomatoes are only rated as good-tasting when both sugar and acid levels are high, since tomatoes with high sugar but low acid concentrations are considered bland (Kader et al., 1978). Citric acid concentrations were higher in EC4 and EC6 as compared to C, giving them the edge over D, which had an even lower citric acid content than C, albeit not to a significant extent (Fig. 2C). Malic acid concentrations on the other hand, were not influenced by the different water regimes (Fig. 2D). 

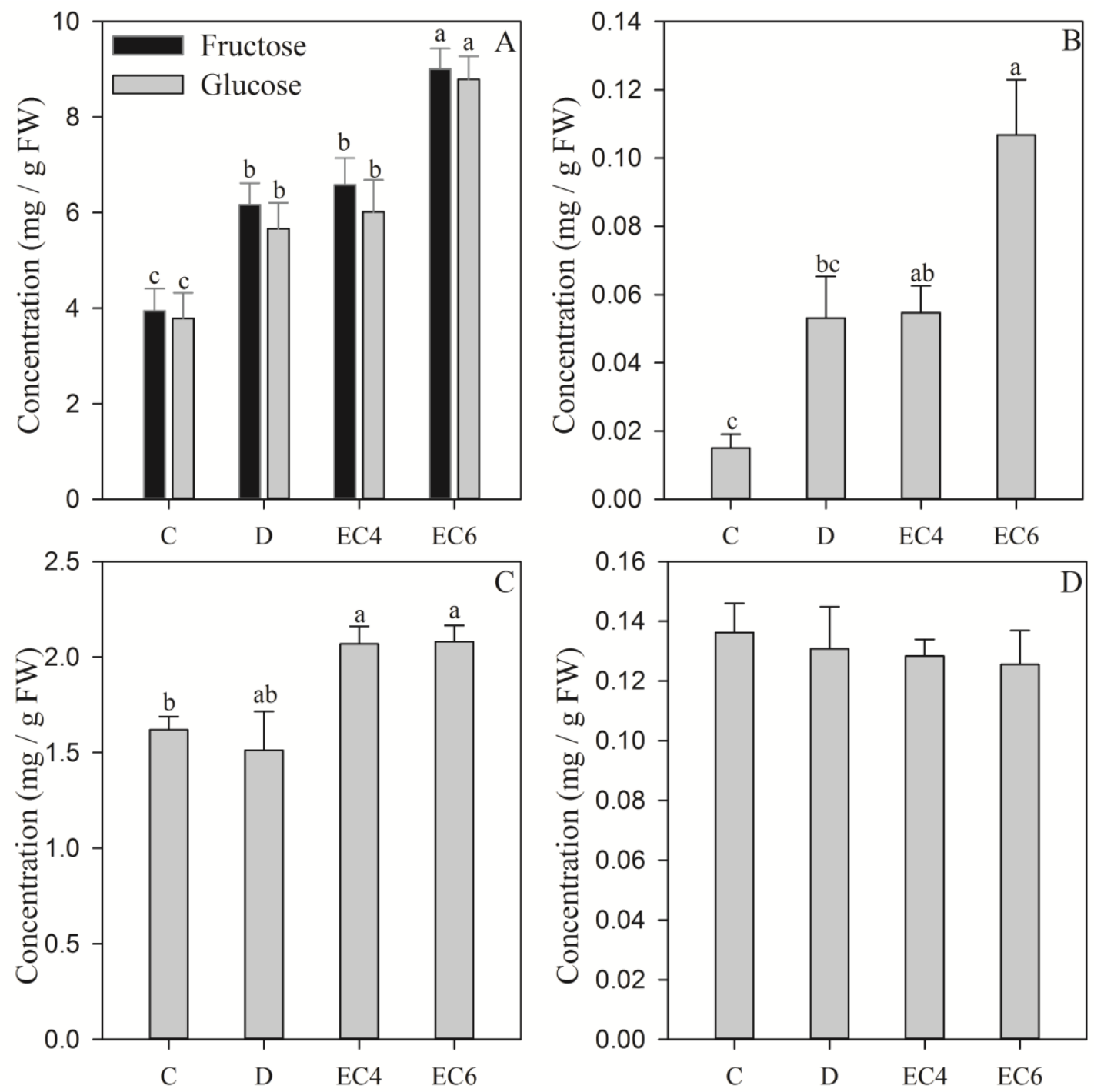

Figure 2. Mean concentrations $( \pm S E, n=18$ ) of the hexose sugars $(A)$ glucose and fructose, and (B) sucrose, and organic acids (C) citric acid and (D) malic acid for the different treatments (C - control, D - drought treatment, EC4 - salt treatment with an EC of $4 \mathrm{dS}$ $\mathrm{m}^{-1}$, EC6 - salt treatment with an EC of $\left.6 \mathrm{dS} \mathrm{m}^{-1}\right)$. Significant differences $(P<0.05)$ across bars are denoted by different letters.

While taste is a crucial quality parameter for obvious reasons, it is surely not the only factor to be considered. Fruit firmness is crucial for the tomato as a commercial product as well, affecting not only organoleptic quality, but also shelf-life and transportability (Ripoll et al., 2014). In analogy with previous findings (Petersen et al., 1998; Sato et al., 2006), we found that increased salinity had a slight but significant positive effect on tomato firmness (Fig. 3). Not many studies have investigated the interrelation between drought and fruit firmness, although it has been suggested that drought can also have a positive effect on this parameter (Ripoll et al., 2014). We observed no significant difference compared to the control treatment (Fig. 3), yet this might be due to the fact that drought was only mild. A more severe drought could hence provide clarity on this matter. 


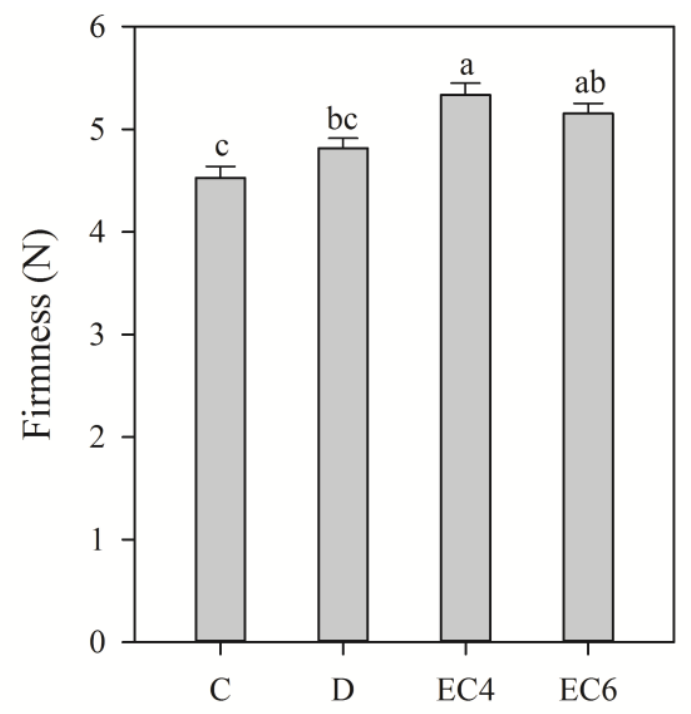

Figure 3. Mean firmness ( $\pm S E, n=216$ ) of the tomatoes for the different treatments ( $C$ - control, $D$ - drought treatment, EC4 - salt treatment with an EC of $4 \mathrm{dS} \mathrm{m}^{-1}$, EC6 - salt treatment with an EC of $\left.6 \mathrm{dS} \mathrm{m}^{-1}\right)$. Significant differences $(P<0.05)$ across bars are denoted by different letters.

Fruit quality parameters are clearly favoured by both drought and increased salinity, the latter showing the most promising results. However, growers are paid per $\mathrm{kg}$ of sold tomatoes, and a higher quality does not necessarily imply a higher price per weight for regular truss tomatoes. It is therefore crucial not to overlook the impact of the imposed treatments on tomato fruit growth, as an indicator of expected yield. In our study, only EC6 showed a significant reduction in diameter and thus fresh weight, whereas D and EC4 did not provoke a water deficit that reduced tomato growth (Fig. 4). However, we do note a slight negative trend in diameter with decreasing $\Psi_{\text {stem }}$. Nonetheless, since quality parameters are affected to a greater extent, the overall balance between production and quality is improved.

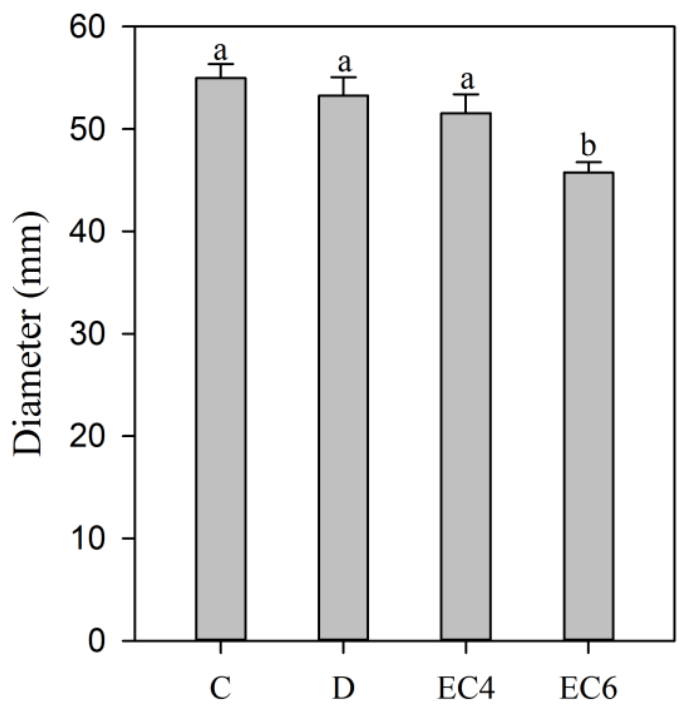

Figure 4. Mean diameter $( \pm S E, n=18)$ of the tomatoes for the different treatments ( $C$ - control, $D$ - drought treatment, EC4 - salt treatment with an EC of $4 \mathrm{dS} \mathrm{m}^{-1}$, EC6 - salt treatment with an EC of $\left.6 \mathrm{dS} \mathrm{m}^{-1}\right)$. Significant differences $(P<0.05)$ across bars are denoted by different letters. 


\section{CONCLUSIONS}

Water deficits, either through diminished water supply or increased salinity, are known to affect multiple aspects of fruit quality. However, yield reduction is mostly reported as being the inevitable price that has to be paid. In this study, it was demonstrated that slight reductions in water supply or slight increases of salinity are already able to positively influence fruit quality significantly, without this necessarily leading to substantial production losses. These insights provide a promising outlook for potential optimisation of both quality and production in greenhouses, especially at the beginning and the end of the growing season, when environmental parameters are not in favour of good quality. Further fine tuning, through a combination of plant measurements and mechanistic modelling, may yield valuable knowledge for improving greenhouse management.

\section{ACKNOWLEDGEMENTS}

The authors thank the Agency for Innovation by Science and Technology in Flanders (IWT) for the PhD funding granted to the first author. The authors are also indebted to Philip Deman, Geert Favyts and Erik Moerman for the technical support, and Veerle De Schepper, Jochen Hanssens, Jesse Seynhaeve, Anne-Sofie Vandenbroucke and Hans Van de Put for their assistance during the experiment.

\section{Literature cited}

Anza, M., Riga, P., and Garbisu, C. (2006). Effects of variety and growth season on the organoleptic and nutritional quality of hydroponically grown tomato. J. Food Qual. 29, 16-37 http://dx.doi.org/10.1111/j.1745-4557.2006.00053.x

Auerswald, H., Schwarz, D., Kornelson, C., Krumbein, A., and Brückner, B. (1999). Sensory analysis, sugar and acid content of tomato at different EC values of the nutrient solution. Sci. Hortic. 82, 227-242 http://dx.doi.org/10.1016/s0304-4238(99)00058-8

Bertin, N., Guichard, S., Leonardi, C., Longuenesse, J.J., Langlois, D., and Navez, B. (2000). Seasonal evolution of the quality of fresh glasshouse tomatoes under mediterranean conditions, as affected by air vapour pressure deficit and plant fruit load. Ann. Bot. 85, 741-750 http://dx.doi.org/10.1006/anbo.2000.1123

Davies, J., and Hobson, G. (1981). The constituents of tomato fruit - the influence of environment, nutrition and genotype. CRC Crit. Rev. Fruit Sci. Nutr. 15, 205-280.

Dorais, M., Papadopoulos, A.P., and Gosselin, A. (2001). Greenhouse tomato fruit quality. In Horicultural Reviews Vol. 26, John Wiley \& Sons, Oxford. p.239-319 http://dx.doi.org/10.1002/9780470650806.ch5

Guichard, S., Gary, C., Leonardi, C., and Bertin, N. (2005). Analysis of growth and water relations of tomato fruits in relation to air vapor pressure deficit and plant fruit load. J. Plant Growth Regul. 24, 201-213 http://dx.doi.org/10.1007/s00344-005-0040-z

Hanssens, J., De Swaef, T., and Steppe, K. (2015). High light decreases xylem contribution to fruit growth in tomato. Plant Cell Environ. 38, 487-498 http://dx.doi.org/10.1111/pce.12411

Ho, L.C., and Hewitt, J.D. (1986). Fruit development. In The Tomato Crop, Chapman and Hall, London. p.201-240 http://dx.doi.org/10.1007/978-94-009-3137-4_5

Kader, A.A., Morris, L.L., Stevens, M.A., and Albright-Holton, M. (1978). Composition and flavor quality of fresh market tomatoes as influenced by some postharvest handling procedures. J. Am. Soc. Hortic. Sci. 103, 6-13

Ke, D., and Boersig, M. (1996). Sensory and chemical analyses of tomato flavours. HortScience 31, 599.

Mitchell, J.P., Shennan, C., and Grattan, S.R. (1991). Developmental changes in tomato fruit composition in response to water deficit and salinity. Physiol. Plant. 83, 177-185 http://dx.doi.org/10.1111/j.1399-3054.1991.tb01299.x

Mizrahi, Y. (1982). Effect of salinity on tomato fruit ripening. Plant Physiol. 69, 966-970 http://dx.doi.org/10.1104/pp.69.4.966

Petersen, K., Willumsen, J., and Kaack, K. (1998). Composition and taste of tomatoes as affected by increased salinity and different salinity sources. J. Hortic. Sci. Biotechnol. 73, 205-215 
Plaut, Z., Grava, A., Yehezkel, C., and Matan, E. (2004). How do salinity and water stress affect transport of water, assimilates and ions to tomato fruits? Physiol. Plant. 122, 429-442 http://dx.doi.org/10.1111/j.13993054.2004.00416.x

Ripoll, J., Urban, L., Staudt, M., Lopez-Lauri, F., Bidel, L.P.R., and Bertin, N. (2014). Water shortage and quality of fleshy fruits-making the most of the unavoidable. J. Exp. Bot. 65, 4097-4117 http://dx.doi.org/10.1093/jxb/eru197

Sato, S., Sakaguchi, S., Furukawa, H., and Ikeda, H. (2006). Effects of $\mathrm{NaCl}$ application to hydroponic nutrient solution on fruit characteristics of tomato (Lycopersicon esculentum Mill.). Sci. Hortic. 109, 248-253 http://dx.doi.org/10.1016/j.scienta.2006.05.003

Veit-Köhler, U., Krumbein, A., and Kosegarten, H. (1999). Effect of different water supply on plant growth and fruit quality of Lycopersicon esculentum. J. Plant Nutr. Soil Sci. 162, 583-588 http://dx.doi.org/10.1002/(sici)15222624(199912)162:6<583::aid-jpln583>3.0.co;2-p 